\title{
Der nekrotische Zerfall der Uterusmyome während der Gravidität.
}

\author{
Von \\ Dr. G. Krukenberg, \\ I. Assistenzarzt an der gynäkologisehen Klinik in Bonn.
}

Nekrotischer Zerfall eines Uterusmyoms ist, zumal im Wochenbette, ein nicht seltenes Vorkommniss. Während der Schwangerschaft dagegen kommt es höchstens zur ödematösen Infiltration. Die Möglichkeit, dass weitergehende Veränderungen auch einmal $\mathrm{zu}$ dieser Zeit entstehen können, soll allerdings nicht geleugnet werden, aber es wird die Thatsache betont, dass - mit Ausnahme einer Beobachtung von Cappie - in allen Fällen, welche dies angeblich beweisen sollen, jene Veränderungen mit grosser Wahrscheinlichkeit erst durch die Wehenthätigkeit oder die Wochenbettsvorgänge herbeigeführt wurden (Gusserow ${ }^{2}$ )). Dass letztere hierzu Veranlassung geben, ist erklärlich; die reichliche Blutzufuhr hingegen, welche die Genitalorgane während der Schwangerschaft erhalten, muss in der That viel eher geeignet erscheinen, ein schnelles Wachsthum der Myome hervorzurufen, als regressive Processe einzuleiten. Auch der vielfach eitirte Fall Cappie's macht nur eine scheinbare Ausnahme. Einmal ist die Beobachtung ungenau (es soll kein Fieber bestanden haben!), so dass wir den Eintritt der Gangrän während der Schwangerschaft auch hier nicht als gesichert betrachten können, ausserdem aber handelte es sich um ein subseröses Myom, dessen Stiel, vermuth-

1) Obstetrical Journal of Gr. Britain 1874, Vol. II, p. 303.

2) Billroth's Handbuch der Frauenkrankheiten, Bd. II, S. 121. 
lich durch einen Fall, 11/2 Mal um seine Achse gedreht war, also um Verhältnisse, für welche die vorhandene Schwangerschaft vollständig gleichgültig war. Eher kann ein Fall Hecker's') hierher gerechnet werden, obgleich während der Schwangerschaft keine Symptome von Seiten der Geschwulst beobachtet wurden. Aber Patientin litt an Lungentuberculose und mag alle Symptome auf diese bezogen haben. Da die spontane Geburt nicht lange dauerte, der Tod schon 6 Stunden post partum erfolgte und bei der Section das interstitielle Myom doch schon Fluctuation und einen centralen, rothen Erweichungsherd zeigte, in welchem nur noch fetzige Gewebsbalken vorhanden waren, so dürfte die Ansicht Hecker's berechtigt sein, dass dieser Herd sich schon im Laufe der Schwangerschaft gebildet habe.

Mit Sicherheit trat Verjauchung eines interstitiellen Uterusmyoms schon während der Schwangerschaft auf in einem Falle, welcher kürzlich in der gynäkologischen Klinik zu Bonn letal endete :

Frau Fr., 43 Jahre alt, früher nie krank, ist seit ihrem 17. Jahre regelmässig und fast schmerzlos menstruirt, seit zwei Jahren steril verheirathet. Auch in den letzten Jahren änderte sich die Periode nur insofern, als sie etwas stärker wurde; zuletzt trat sie Anfang März auf. Anfang April bemerkte Patientin, dass der Leib etwas stärker war als sonst, und erklärte sich dies, zumal Beschwerden nicht vorhanden waren, aus eingetretener Schwangerschaft. Erst am 10. Juni stellten sich im unmittelbaren Anschlusse an eine angeblich nicht beschwerliche Defäcation plötzlich ein fünf Minuten lang anhaltender Schüttelfrost ein, verbunden mit den heftigsten Schmerzen über der Symphysis, derenthalben Patientin nur gebüickt und mit grosser Anstrengung bis in ihr Zimmer gehen konnte. Sie legte sich sofort zu Bette. Der behandelnde Arzt erklärte das Leiden für eine Unterleibsentzündung (Blutegel auf das rechte Hypogastrium). Nach zwei Tagen liessen die Schmerzen etwas nach, blieben jedoch immer noch so stark, dass Patientin bis Ende Juni das Bett hüten musste. Die Erscheinungen waren die einer rechtsseitigen Pelveoperitonitis. Die Schmerzen sassen meistens rechts und strahlten in das rechte Bein aus. Stuhlgang und Urinentleerung waren anfangs stark schmerzhaft und wurden erst später schmerzlos; nie Brechen, nie Ohnmachten oder Schwindel. Der Leibesumfang nahm stetig zu. Von Ende Juni an fühlte sich Patientin vorübergehend etwas besser, stand gelegentlich auf, hatte jedoch immer noch Schmerzen in der rechten Unterleibsgegend und im rechten Beine. Als die Beschwerden von neuem zunahmen, suchte Patientin die Klinik auf.

1) Klinik der Geburtskunde, Bd. II, S. 128. 
Status praesens (20. Juli):

Leidlich genährte, etwas blasse Frau. Herz und Lungen vollständig normal. Leber und Milz etwas nach oben gedrängt, sonst normal. Das Abdomen ist in seinen unteren Partien aufgetrieben, rechts etwas stärker als links. Die fast schmerzlose Palpation und Percussion ergiebt die Abwesenheit von Ascites, und ferner, dass sich in den unteren Partien des Abdomen zwei Tumoren befinden, von welchen der rechte, bis zur Nabelhöhle heraufreichende, hart ist, während der linke, zwei Querfinger breit weniger nach oben ragende, sich prall-elastisch anfuihlt. Sie sind in ihren oberen Theilen durch eine längsverlaufende, ziemlich median im Abdomen gelegene, aber nur oberflächliche Furche getrennt, während eine solche weiter unten nicht mehr mit Sicherheit zu fühlen ist. Beide sind glatt, nicht böckerig. Einen dritten, bedeutend kleineren, etwa wallnussgrossen Tumor fühlt man ganz median, dicht über der Symphysis der Tumormasse breit und ziemlich unbeweglich aufliegend; er ist von harter Consistenz und leicht höckeriger Oberläche. Bei der Auscultation gelingt es ein Mal über dem rechten Hypogastrium deutliches Uteringeräusch zu hören. Kindliche Herztöne sind nicht wahrnehmbar. Bei der internen und bimanuellen Untersuchung ergiebt sich, dass die ziemlich weit hinten stehende, ziemlich hochgelegene und anfgelockerte Portio allmälig in die einheitlich erscheinende Tumormasse übergeht. Zuweilen fühlt man links undeutliches Ballotement. Die Rectalpalpation weist im rechten Parametrium einzelne härtere Stränge nach. Ovarien und Ligamenta rotunda nicht zu fühlen.

Herr Geh. Rath Veit hielt bei diesem Befunde eine sichere Diagnose für unmöglich. Mit Wahrscheinlichkeit handelte es sich um Gravidität im fünften bis sechsten Monate. Extrauterine Schwangerschaft liess sich ziemlich sicher ausschliessen. Wahrscheinlich war der linksseitige Tumor der gravide Uterus, der rechtsseitige ein Myom. Aber gegen diese, auf Grund der Untersuchung gemachte Annahme sprach der bisherige Krankheitsverlauf. Wie sollte, nach den bisherigen Erfahrungen, durch ein interstitielles oder doch jedenfalls breitbasig aufsitzendes Myom schon im vierten Schwangerschaftsmonate eine offenbar sehr acute und intensive Entzündung herbeigeführt worden sein? Der Anamnese nach musste man viel eher an einen Ovarialtumor denken, welcher - sei es durch Torsion des Stieles, sei es ohne solche - zur Vereiterung gekommen war. Aber diese Annahme liess sich wieder mit dem Befunde nicht vereinigen. Ovarialcys tome können allerdings einen sehr engen Zusammenhang mit dem graviden Uterus zeigen ${ }^{1}$ ); aber, nahm man an, dass der rechtsseitige

1) OIshausen, Krankheiten der Ovarien. S. 102. 
Tumor rom Ovarium ausging, so musste es seiner Consistenz nach ein fester sein, und in diesem Falle musste man eine deutliche trennende Furche erwarten, die hier besonders in der unteren Partie fehlte. Auch liess sich bei der Härte des rechtsseitigen Tumors nicht gut annehmen, dass er in Vereiterung begriffen sei, und ohne solche Annahme war der Krankheitsverlauf wieder nicht erklärlich. Nahm man dagegen an, dass der linksseitige, prall-elastische Tumor ein vereitertes Ovarialcystom sei, der rechtsseitige, über dem Uteringeräusche gehört wurde, der gravide Uterus, so war besonders nicht erklärt, warum die Entzündung rechts localisirt war.

Erst am ersten August ergab eine nochmalige Untersuchung sichere Anhaltspunkte für die Diagnose. Die Lagerungs- und Grössenverhältnisse waren jetzt verändert. Der linksseitige Tumor ragte bis zur Nabelhöhle, der rechtsseitige war nicht höher gerückt. Die dem linksseitigen Tumor eigenthümliche Resistenz hatte sich in der unteren Partie weiter nach rechts verbreitert, um demnach erst rechts von der Linea alba in die härtere Resistenz des rechtsseitigen Tumors überzugehen. Auch in den oberen Partien war eine Trennungsfurche nicht mehr vorhanden. Der kleine, über der Symphysis fühlbar gewesene Tumor lag, sonst unverändert, ein wenig höher und weiter nach links dem linksseitigen Tumor auf. Ausserdem hörte man rechts und besonders links häufiges Uteringeräusch und in der Linea alba - also noch im Bereiche des linksseitigen Tumors - fötale Herztöne und fühlte bei Druck auf den linksseitigen Tumor den Kopf über der Portio vaginalis ballotirend.

War somit der linksseitige Tumor der gravide Uterus, so blieb nur noch die Deutung des rechtsseitigen Tumors übrig. Offenbar waren von ihm aus die Krankheitssymptome ausgegangen. Es muss hier eingeschaltet werden, dass Patientin, welcher dauernde Bettlage verordnet war, in ihrem subjectiven Befinden nicht die geringste Veränderung verspürte; ihre Klagen waren noch dieselben. Die Temperatur war Morgens normal oder leicht febril, Abends war meist höheres Fieber, bis 39,9, vorhanden; irgend eine Regelmässigkeit des Fiebers liess sich nicht erkennen. Gelegentlich war in den unteren Lungenlappen leichtes Rasseln nachzuweisen, nie eine-Dämpfung. Bei dem unveränderten Befinden der Patientin musste man annehmen, dass die Krankheit von Anfang an mit Fieber verlaufen war. Der ganz unregelmäs- 
sige Typus desselben liess - bei normalem Verhalten der anderen Organe und den stets rechts unten im Abdomen localisirten Schmerzen - an einen dort befindlichen Eiterungsprocess denken. Auch jetzt war der Untersuchungsbefund durch die Annahme eines vereiterten Ovarialtumors nicht zu erklären. Gegen einen rechtsseitigen, im subperitonealen Bindegewebe entwickelten Abscess sprach die Härte und fehlende Fluctuation des Tumors, doch liess sich diese Annahme nicht ausschliessen. Eine zweite Möglichkeit war die, dass es sich um ein vereitertes Myom, eine dritte die, dass es sich um Eiterretention in einem rudimentären Nebenhorne handele. Eine sichere Differentialdiagnose blieb unmöglich, aber für die Annahme eines Myomes sprach sehr entschieden die beobachtete Abnahme der Trennungsfurche ${ }^{1}$ ) (sogenannte Abflachung).

In der folgenden Zeit trat keine merkliche Veränderung am Abdomen ein, nur dass die kindlichen Herztöne, welche am 8. August noch zu hören waren, sich vom 10. August an nicht mehr auffinden liessen. Die Fiebercurve blieb unregelmässig, wie zuvor; bald war weder am Morgen noch am Abend Fieber vorhanden, bald nur Abends (bis 40,7), bald auch Morgens (bis $39,0)$. Patientin, die schon früher bei abendlichen Temperatursteigerungen leichte Fröste gehabt hatte, bekam wiederholt stärkere Fröste und collabirte zusehends. Das Sensorium war zuweilen benommen, die Pulse wurden unregelmässig und klein $(110-120)$; die Zunge trocken, leicht borkig; Haut und Schleimhäute zuweilen cyanotisch. Urin trübe und an Menge gering. Patientin konnte sich bald nicht mehr aufrichten; am 10. August wurde ein ganz oberflächlicher Decubitus am Kreuzbeine constatirt.

Am 11. August Nachmittags trat ein sehr intensiver Schüttelfrost ein; eine Stunde spätex betrug die Temperatur 41,1. Eine genaue nochmalige Untersuchung ergab dieselben Verhältnisse wie zuvor. Die Portio vaginalis war verkürzt, der Muttermund geschlossen; es ging ziemlich reichlich nicht riechender, gelblicher Schleim ab. Nur wenn es gelang, den supponirten Eiterherd zu eröffnen, erschien es möglich, die Patientin am Leben zu erhalten. Dieser Eiterherd musste nach obigen Deductionen in der vormals noch durch eine Furche vom Uterus getrennten rechtsseitigen Härte gelegen sein. Da letztere während der Beobachtungszeit nicht nach oben gestiegen war, da ferner peritonitische Erschei-

1) Spiegelberg, Monatsschrift für Geburtskunde, Bd. XXVIII, S. 428. 
nungen rechts vorhanden gewesen waren, so durfte man hoffen, dass diese Stelle mit dem Peritoneum parietale verwachsen sei, dass es also gelingen würde, beim Einschnitte an dieser Stelle nicht nur den Eiterherd zu treffen, sondern auch dem Eiter ohne Eröffnung der Peritonealhöhle Abfluss zu verschaffen.

Herr Geh. Rath Veit schritt deshalb am 12. August Morgens $6 \mathrm{Uhr}$ unter antiseptischen Cautelen zur Probeincision: Narkose. $12 \mathrm{~cm}$ langer, längs verlaufender Hautschnitt in der Mitte zwischen Nabel und Spina anterior superior dextra direct auf die rechtsseitige Härte. Die Erwartung, in welcher diese Probeincision gemacht war, wurde vollständig getäuscht. Die Peritonealhöhle ist an der Incisionsstelle nicht obliterirt, nicht einmal strangförmige Adhäsionen sind vorhanden. Es entleeren sich nur einige Esslöffel klarer, gelblicher Flüssigkeit. Ein rechtsseitiger und ein linksseitiger Tumor ist nicht $\mathrm{zu}$ unterscheiden, die ganze Tumormasse imponirt vielmehr als gravider Uterus, der zwar rechts und oben etwas härter ist als sonst, aber keine Verfärbung und überhaupt nichts Krankhaftes erkennen lässt. Der in das Peritonealcavum eingeführte Zeigefinger kann bis auf den früher erwähnten dritten Tumor, ein kleines Fibrom, nichts $\mathrm{Ab}$ normes entdecken. Diag n os is va cat. Schluss der Bauchwunde. Heftpflaster - Watteverband.

Abends 6 Uhr war die Körpertemperatur nach einem starken Schweisse auf 35,5 (Vaginalmessung), die Pulsfrequenz auf 90 gefallen! Abends $7 \mathrm{Uhr}$ Wehenbeginn, $11 \mathrm{Uhr}$ schnelle Geburt eines Fötus in vollständig geschlossenen Eihäuten. Placenta und Eihäute ohne Besonderheit; das Fruchtwasser nur leicht getrübt, nicht riechend; der weibliche Fötus vollständig normal gebildet, frischtodt, nicht macerirt. (Gewicht $=520 \mathrm{~g}$, Länge $=30 \mathrm{~cm}$.) - Das Abdomen war jetzt etwas aufgetrieben und empfindlich, die bimanuelle Exploration ausserdem durch den Verband erschwert; nach rechts und oben vom Uterus fühlte man, mit ihm eng zusammenhängend, einen faustgrossen, harten Tumor. Weder vor noch während oder nach der Geburt fand ein abnormer Blutabgang oder Eiterentleerung statt.

In den folgenden Tagen wurde der Leib stark aufgetrieben und sehr empfindlich. Patientin erbrach zuweilen, war zeitweise klar und klagte dann viel über Schmerzen, meist war sie in leichten Delirien befangen. Am 14. August trat Herpes labialis auf, am 16. August linksseitige Parotitis. Der Urin blieb trübe 
und musste seit der Operation mittels Katheter entleert werden, in den letzten zwei Tagen ging er unwillkürlich ab. Das Lochialsecret war riechend, nie besonders reichlich, nie mit Eiter ge mengt. Vom 16. August an bestand vollständiger Status typhosus.

Im Gegensatze zu dieser Verschlechterung im Zustande der Patientin blieben Temperatur (Vaginalmessungen, die in den ersten Tagen von mir selbst controlirt wurden) und Pulsfrequenz niedriger als zuror, um sich erst in den letzten Tagen wieder zu heben.

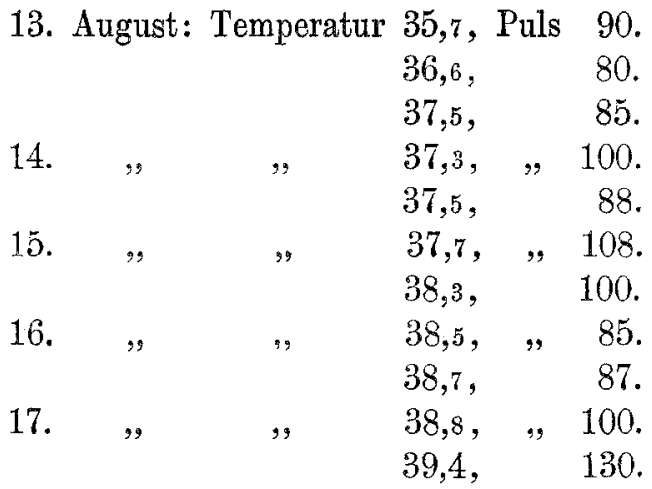

Tod am 18., Morgens 4 Uhr.

Section 8 Stunden post mortem (Dr. Ribbert). Auszug aus dem Sectionsprotokolle: Gehirn ohne Besonderheiten. In der linken Parotis miliare gelbliche Herde. Herz und Lungen normal. Aus dem Abdomen entleert sich eitrige Flüssigkeit. Sämmtliche Darmschlingen sind unter einander durch gelbeitrig-fibrinöse Massen verklebt, wie solche auch das Peritoneum parietale und die Leber bedecken; sonst Magen und Darmkanal ohne Veränderung. Die Nieren mit meist unter linsengrossen, graugelblichen Herden durchsetzt. Nierenbecken geröthet, nur rechterseits etwas erweitert. Ureteren, kleinfingerdick, enthalten trüben Urin.

Aus dem Becken ragt ein etwas über kindskopfgrosser, mit eitrig-fibrinösen Massen nur wenig bedeckter, glatter Tumor hervor, auf welchem vorn ein wallnussgrosses, hinten ein kirschengrosses, nicht erweichtes Myom prominirt. Im Uebrigen erscheint der Tumor annähernd kugelig, ohne jede besondere Prominenz. Ovarien und Tuben hyperämisch, sonst ohne Veränderung; im linken Ovarium ein Corpus luteum. Beim Aufheben des Tumors ergiebt sich, dass er rechts und hinten in der Ausdehnung von etwa einer Handfläche des ihn sonst überziehenden Peritoneum 
beraubt ist. Das gegenüberliegende Peritoneum der rechten seitlichen Beckenwand (Ligamentum latum) und das den Douglas'schen Raum in seiner rechten Hälfte bildende Peritoneum fehlen ebenfalls. Der Theil des Tumors und das Zellgewebe, welches dadurch blossgelegt ist, sind in eine graue, schmutzig -schmierige, halbflüssige Masse verwandelt und bilden zusammen einen rechts und hinten im kleinen Becken gelegenen, nach der Peritonealhöhle zu weit offenen Hohlraum, in welchem der innere Rand des Psoas, die Vena iliaca externa und der Nervus obturatorius stellenweise freiliegen.

Klarer werden die Verhältnisse nach Herausnahme der Beckenorgane: Harnblase contrahirt, nur wenig trüben Urin enthaltend; ihre Schleimhaut katarrhalisch ecchymosirt. Cervix uteri sehr weit, auf seiner Innenfläche grauschwarz gefärbt. Der puerperal veränderte Uterus liegt in der linken Seite des beschriebenen Tumor. Seine Wandung ist links gut daumendick, rechts nimmt sie ein interstitiell gelegenes, kugeliges, etwa $8 \mathrm{~cm}$ im Durchmesser haltendes Myom in sich auf. Die Kapsel desselben ist durchschnittlich $1-1 \frac{1}{2} \mathrm{~cm}$ dick, rechts und hinten jedoch verdünnt sie sich und ist an der Stelle des oben beschriebenen Defectes vollständig zu Grunde gegangen. In diesem Defecte liegt also das Myom selbst in grosser Ausdehnung bloss und ist hier, während es soust röthlich und nur wenig erweicht, bis zu $3 \mathrm{~cm}$ Tiefe in graue, schmutzig-schmierige Masse verwandelt. Weiter nach unten ist die Kapsel wieder vorhanden, liegt aber hier dem Myom nicht dicht an, vielmehr setzt sich hier die oben erwähnte fetzige Höhle in einen ebenso beschaffenen, einerseits vom Myom, andererseits von dessen Kapsel gebildeten schmalen Spalt fort, welcher sich mit markstückgrosser, fetziger Oeffnung rechts etwas oberhalb des Orificium internum in das Uteruscavum öffnet. Diese Oeffnung wird zum Theil durch die angrenzenden Partien des Myoms verlegt.

Der Tumor wurde als Myom bezeichnet, weil er mikroskopisch zahlreiche Muskelfasern aufwies; im Uebrigen bot er keine Besonderheiten.

Die Verjauchung war so hochgradig, dass sich das Präparat nicht conserviren liess und schon am folgenden Tage jede weitere Untersuchung unmöglich war.

Der Krankheitsverlauf ist danach verständlich: Im vierten Schwangerschaftsmonate traten Ernährungsstörungen und Rei- 
zungen in einem interstitiell entwickelten Myom auf. Man wird nicht irren, wenn man diese Störungen, welche acut im unmittelbaren Anschlusse an eine Defäcation entstanden (zur Zeit, wo sonst die Periode einzutreten pflegte?), auf eine Blutung zwischen das Myom und seine Kapsel zurückf ührt. Weiterhin kam es zu einer Verjauchung der Kapsel und der Oberfläche des eigentlichen Myoms. Nach Verjauchung der Kapsel brach der Herd in das rechte Parametrium durch, unterwühlte das Zellgewebe, legte schliesslich Nerven und Gefässe frei, ohne jedoch vorläufig den peritonealen Ueberzug des Parametrium zu durchbrechen; die Symptome blieben daher noch die einer circumscripten Jauchung und Jaucheresorption. Erst die Geburtswehen brachten mechanisch den Durchbruch zu Stande und riefen plötzlich in der noch kurz vorher bei der Probeincision gesund gefundenen Peritonealhöhle eine schwere eitrige Entzündung hervor. Auch in die Uterushöhle brach der Herd durch. Dies geschah möglicherweise gleichfalls durch die Wehen resp. die Entleerung des Uterus; wahrscheinlicher ist es indessen, dass der Causalnexus der umgekehrte war, dass also der Jauchehord schon vor der Geburt sich an einer kleinen Stelle der Uterushöhle genähert hatte, durchbrach und nun die Wehen veranlasste. Dass der Eintritt der Geburt durch die Operation hervorgerufen wurde, ist nicht sehr wahrscheinlich, weil schon vorher die Portio vaginalis verkürzt war und reichlich gelblicher Schleim abging.

Die Diagnose scheiterte einmal deshalb, weil eine derartige Veränderung eines interstitiellen Myoms während der Schwangerschaft bisher nicht beobachtet ist, dann aber auch deshalb, weil sich der ganze Process an der hinteren, dem Darm - und Kreuzbeine zugekehrten Seite des Tumors vollzog, der physikalischen Untersuchung daher selbst bei der Incision verborgen blieb. Die anamnestischen Angaben und die Symptome wie bei Vereiterung eines Ovarialtumors, verbunden mit der a uffallenden Schmerzlosigkeit der Palpation, ein damit nicht vereinbarer, vielmehr zur Annahme eines Uterasmyoms drängender Befund, speciell eine allmälige Abflachung des Tumors, sind die Momente, welche in Zukunft die Diagnose ermöglichen werden.

Gesetzt der Abort, dessen Eintritt hier möglicherweise nur durch die dauernde Bettlage verzögert wurde, wäre schon einen Monat früher eingetreten, so wäre der Tod vielleicht erst so spät nach der Geburt erfolgt, dass auch in diesem Falle der 
Nachweis, die Verjauchung rühre aus der Gravidität her, unmöglich gewesen wäre. Wenn die Veränderungen der Myome schon in ihrem Beginne, ehe sie klinisch erkennbar sind, Abort herbeiführen, so wird es erklärlich, warum sie fast nie auf die Schwangerschaft, sondern auf die Geburt oder das Wochenbett zurückgeführt werden. Auf Grund dieser Erwägung lassen wir es unentschieden, sowohl ob nekrotischer Zerfall der Myome wirklich so überaus selten in der Schwangerschaft entsteht, wie man neuerdings annimmt, als auch ob zwischen Schwangerschaft und nekrotischem Zerfalle ein causaler Zusammenhang besteht oder nicht.

Zur Pflege der Patientin war auch eine bisher stets gesunde Schwangere verwandt worden. Letzte Periode Ende December. Dieselbe erkrankte am 1. September mit heftigem Schüttelfrost (Temperatur $=39,5)$ und gab jetzt an, dass sie sich schon während der Pflege der Frau Fr. unwohl gefühlt, an Appetitlosigkeit und Frösteln gelitten habe. Es entwickelte sich ein ganz unregelmässig intermittirendes Fieber (bis 40,2), welches gelegentlich von Schüttelfrösten begleitet war und mit tagelangen Unterbrechungen bis zum 16. October anhielt. Die physikalische Untersuchung ergab keinen positiven Anhalt dafür; nur ein Mal (13. September) liess sich unterhalb der rechten Scapula eine leichte Abschwächung des Percussionsschalles constatiren. Vom 5. bis 13. September bestand starker Herpes labialis bei freiem Sensorium, vom 9 . bis 13. September blutige Diarrhoen. Am 20. September erfolgte die leichte Geburt eines lebenden und gesunden Knaben (Gewicht 1810, Länge = 44). Placenta normal. Das atypische Fieber wurde durch den Eintritt der Geburt nicht erkennbar beeinflusst. Andererseits liess sich auch im Wochenbette an den Genitalorganen bis auf einen unbedeutenden Geruch der Lochien nichts Abnormes auffinden. Am 4. November wurde die Wöchnerin gesund entlassen.

Es ist im hohen Grade wahrscheinlich, dass sie bei der Pflege der Frau Fr. inficirt wurde. Bemerkenswerth ist, dass auch bei ihr Herpes auftrat, wie solcher überhaupt bei der sogenannten kryptogenetischen Septico-Pyämie auffallend häufig beobachtet worden ist. ${ }^{1}$ ) Bemerkenswerth ist ferner, dass das Fieber in beiden Fällen ein höchst unregelmässig intermittirendes war.

1) Leube, Dentsches Archiv für klinische Medicin, XXII, S. 249. P. Wagner, Ibid., XXVIII, S. 539. - Pel, Zeitschrift für klinische Medicin, IV, S. 564. 